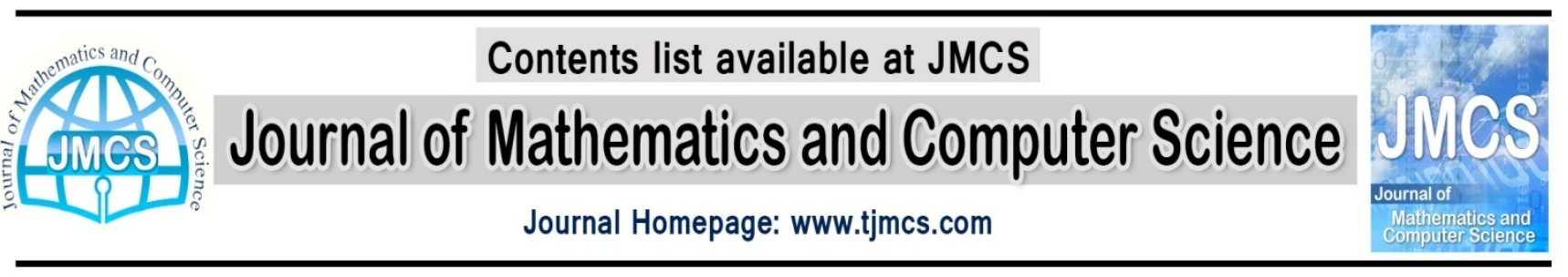

\title{
FEW COMMON FIXED POINT RESULTS FOR WEAKLY COMMUTING MAPPINGS
}

\author{
Amalendu Choudhury \\ Dept. of Mathematics and Statistics, Haflong Govt. College \\ Haflong, Dima Hasao-788819, India \\ E-mail: choudhury.amalendu@yahoo.com
}

T. Som

Dept. of Applied Mathematics, Indian Institute of Technology

(Banaras Hindu University)

Varanasi - 221005, India

E-mail:som_tanmoy@yahoo.co.in

Article history:

Received March 2011

Accepted December 2012

Available online January 2013

\begin{abstract}
In this paper we give a common fixed point result for a mapping of f-contractive mappings, which generalize the results of Chang [2], Hadzic [4] and Sessa et.al [10]. We also extend the results of Fisher [3], Imdad and Khan [7], Iseki [6], Singh [11], Singh and Tiwari [12].
\end{abstract}

Key words : Weakly commuting maps, f-contraction, common fixed point.

\section{Introduction}

Let $\mathrm{T}$ be a mapping of a nonempty set $\mathrm{X}$ into itself . Then $\mathrm{T}$ is said to have a fixed point if there exists a point $\mathrm{u}$ in $\mathrm{X}$ such that $\mathrm{Tu}=\mathrm{u}$.

Two self mappings $A$ and $S$ of a metric space $(X, d)$ are called weakly commuting if

$$
\mathrm{d}(\mathrm{ASx}, \mathrm{SAx}) \leq \mathrm{d}(\mathrm{Ax}, \mathrm{Sx}) \text { for all } \mathrm{x} \in \mathrm{X} \text {. }
$$

Let $(\mathrm{X}, \mathrm{d})$ be a complete metric space, $\mathrm{N}$ the set of all positive integer and let $\mathrm{F}$ be the set of all real functions $f:[0, \infty) \rightarrow[0, \infty)$ such that every $f \in F$ is non decreasing, upper semi continuous and $f(t)<t$ for any $\mathrm{t}>0$.

For a family of mappings Hadzic [4] proved the following result. 
Theorem 1.1 Hadzic [4] Let $S, T: X \rightarrow X$ be two continuous mappings and $\left\{A_{i}\right\}_{i \in N}$ be a family of self mappings of $X$ satisfying

(i) $\quad A_{i}(X) \subseteq S(X) \cap T(X)$ for each $i \in N$.

(ii) $\quad A_{i}$ commutes with $S$ and $T$ for each $i \in N$.

(iii) $d\left(A_{i} x, A_{j} y\right) \leq q d(S x, T y)$ for any $x, y \in X$ and $i, j \in N, i \neq j$, where $0 \leq q<1$. Then $\left\{A_{i}\right\}_{i \in N}$,

$\mathrm{S}$ and $\mathrm{T}$ have a unique common fixed point.

Generalizing the results of Husain and Sehgal [5] and Iseki [6], Chang [2] established the following result for a family of f-contraction mappings :

Theorem 1.2 [2] Let $S, T: X \rightarrow X$ be two continuous maps. Then $S$ and $T$ have a common fixed point $\mathrm{w}$ if and only if there exists two self mappings $\mathrm{A}, \mathrm{B}$ of $\mathrm{X}$ and a function $\mathrm{f} \in \mathrm{F}$ such that

(iv) $\mathrm{A}(\mathrm{X}) \cup \mathrm{B}(\mathrm{X}) \subseteq \mathrm{S}(\mathrm{X}) \cap \mathrm{T}(\mathrm{X})$

(v) both $\mathrm{A}$ and $\mathrm{B}$ commute with $\mathrm{S}$ and $\mathrm{T}$,

(vi) $\mathrm{d}(\mathrm{Ax}, \mathrm{By}) \leq \mathrm{f}(\max \{\mathrm{d}(\mathrm{Sx}, \mathrm{Ty}), \mathrm{d}(\mathrm{Sx}, \mathrm{Ax}), \mathrm{d}(\mathrm{Ty}, \mathrm{By}),[\mathrm{d}(\mathrm{Sx}, \mathrm{By})+\mathrm{d}(\mathrm{Ty}, \mathrm{Ax})] / 2\})$,

for any $x, y \in X$. Further $w$ is the unique common fixed point of $A, B, S$ and $T$.

Unifying the above results of Hadzic [4] and Chang [2], Sessa et.al. [10] proved two common fixed point results for weakly commuting mappings. We further generalize the condition satisfied by the mappings and obtain some new results. Before giving our main result we prove few lemmas.

Lemma 1.1 (Chang [2]) Let $f \in F$ and $t_{o}>0$. If $t_{n+1} \leq f\left(t_{n}\right)$ for $n \in N$, then the sequence $\left\{t_{n}\right\}_{n \in N}$ converges to zero.

Proof. See Chang [1].

Let $\mathrm{S}, \mathrm{T}:(\mathrm{X}, \mathrm{d}) \rightarrow(\mathrm{X}, \mathrm{d})$ and $\left\{\mathrm{A}_{\mathrm{i}}\right\}_{\mathrm{i} \in \mathrm{N}}$ be a family of self mappings of $\mathrm{X}$ satisfying condition (i) and the following generalized inequality (4)

(4) $\operatorname{ad}\left(\mathrm{A}_{\mathrm{i}} \mathrm{x}, \mathrm{A}_{\mathrm{j}} \mathrm{y}\right)+\mathrm{bd}\left(\mathrm{Ty}, \mathrm{A}_{\mathrm{j}} \mathrm{y}\right)-\min \left\{\mathrm{d}\left(\mathrm{Ty}, \mathrm{A}_{\mathrm{i}} \mathrm{x}\right), \mathrm{d}\left(\mathrm{Sx}, \mathrm{A}_{\mathrm{i}} \mathrm{x}\right)\right\}$

$$
\leq f\left(\max \left\{d(S x, T y), d\left(S x, A_{i} x\right), d\left(T y, A_{j} y\right), d\left(T y, A_{i} x\right),\left[d\left(S x, A_{j} y\right)+d\left(T y, A_{i} x\right)\right] / 2\right\}\right)
$$

for all $x, y \in X, i, j \in N, i \neq j$, where $f \in F$ and $a+b \geq 1$. Then for any arbitrary point $x_{0} \in X$, we get a point $x_{1} \in$ $X$, guaranteed by (i), such that $\mathrm{Tx}_{1}=\mathrm{A}_{1} \mathrm{x}_{0}$ and for this $\mathrm{x}_{1}$, there exists a point $\mathrm{x}_{2} \in X$ such that $\mathrm{Sx}_{2}=\mathrm{A}_{2} \mathrm{x}_{1}$ and so on. Inductively we get a sequence $\left\{\mathrm{y}_{\mathrm{n}}\right\}_{\mathrm{n} \in \mathrm{N}}$ such that

$$
\begin{aligned}
& y_{2 n+1}=T x_{2 n+1}=A_{2 n+1} x_{2 n} \text { for every } n \in N_{o}=N \cup\{0\} . \\
& y_{2 n}=S x_{2 n}=A_{2 n} x_{2 n-1} \text { for every } n \in N .
\end{aligned}
$$

Let $d_{n}=d\left(y_{n}, y_{n+1}\right)$ for every $n \in N$. Consequently the following two lemmas also hold.

Lemma 1.2: The sequence $\left\{\mathrm{d}_{\mathrm{n}}\right\}_{\mathrm{n} \in \mathrm{N}}$ converges to zero.

Proof : Using (4), we have for $n \in \mathrm{N}_{\mathrm{o}}$. $\operatorname{ad}\left(A_{2 n+1} x_{2 n}, A_{2 n+2} x_{2 n+1}\right)+b d\left(\operatorname{Tx}_{2 n+1}, A_{2 n+2} x_{2 n+1}\right)-\min \left\{d\left(\operatorname{Tx}_{2 n+1}, A_{2 n+1} x_{2 n}\right), d\left(\operatorname{Sx}_{2 n}, A_{2 n+1} X_{2 n}\right)\right\}$ 


$$
\begin{aligned}
& \leq \mathrm{f}\left(\operatorname { m a x } \left\{\mathrm{d}\left(\mathrm{Sx}_{2 \mathrm{n}}, \mathrm{Tx}_{2 \mathrm{n}+1}\right), \mathrm{d}\left(\mathrm{Sx}_{2 \mathrm{n}}, \mathrm{A}_{2 \mathrm{n}+1} \mathrm{x}_{2 \mathrm{n}}\right), \mathrm{d}\left(\mathrm{Tx}_{2 \mathrm{n}+1}, \mathrm{~A}_{2 \mathrm{n}+2} \mathrm{X}_{2 \mathrm{n}+1}\right), \mathrm{d}\left(\mathrm{Tx}_{2 \mathrm{n}+1}, \mathrm{~A}_{2 \mathrm{n}+1} \mathrm{x}_{2 \mathrm{n}}\right),\right.\right. \\
& \left.\left[\mathrm{d}\left(\mathrm{Sx}_{2 \mathrm{n}}, \mathrm{A}_{2 \mathrm{n}+2} \mathrm{X}_{2 \mathrm{n}+1}\right)+\mathrm{d}\left(\mathrm{Tx}_{2 \mathrm{n}+1}, \mathrm{~A}_{2 \mathrm{n}+1} \mathrm{x}_{2 \mathrm{n}}\right) / 2\right\}\right) \\
& \text { i.e., } \quad \operatorname{ad}_{2 \mathrm{n}+1}+\mathrm{bd}_{2 \mathrm{n}+1}-\min \left\{0, \mathrm{~d}_{2 \mathrm{n}}\right\} \leq \mathrm{f}\left(\max \left\{\mathrm{d}_{2 \mathrm{n}}, \mathrm{d}_{2 \mathrm{n}}, \mathrm{d}_{2 \mathrm{n}+1}, 0,\left(\mathrm{~d}_{2 \mathrm{n}}+\mathrm{d}_{2 \mathrm{n}+1}\right) / 2\right\}\right) \\
& \text { i.e., } \quad(\mathrm{a}+\mathrm{b}) \mathrm{d}_{2 \mathrm{n}+1} \leq \mathrm{f}\left(\max \left\{\mathrm{d}_{2 \mathrm{n}}, \mathrm{d}_{2 \mathrm{n}+1},\left(\mathrm{~d}_{2 \mathrm{n}}+\mathrm{d}_{2 \mathrm{n}+1}\right) / 2\right\}\right) \\
& \text { We claim that } d_{2 n+1}<d_{2 n} \text {. If } d_{2 n+1}>d_{2 n} \text { for some } n \in N_{o} \text {, then }(a+b) d_{2 n+1} \leq f\left(d_{2 n+1}\right) \\
& \text { i.e., } \quad d_{2 n+1} \leq f\left(d_{2 n+1}\right) /(a+b)<f\left(d_{2 n+1}\right) \text {, a contradiction to the property of } f \text {. Thus we get } \\
& \text { (7) } \quad d_{2 n+1} \leq d_{2 n} \text { and }(a+b) d_{2 n+1} \leq f\left(d_{2 n}\right) \text { i.e., } d_{2 n+1} \leq f\left(d_{2 n}\right) /(a+b) \leq f\left(d_{2 n}\right) \quad \text { for every } n \in N_{0} \text {. }
\end{aligned}
$$

Similarly, we can show that

$$
d_{2 n+2} \leq d_{2 n+1} \text { and } d_{2 n+2} \leq f\left(d_{2 n+1}\right) \quad \text { for every } n \in N_{o} \text {. }
$$

From (7) and (8) we find that $\left\{d_{n}\right\}_{n \in N}$ is a decreasing sequence such that $d_{n+1} \leq f\left(d_{n}\right)$ for every $n \in N_{o}$. Then by Lemma 1.1, the sequence $\left\{\mathrm{d}_{\mathrm{n}}\right\}_{\mathrm{n} \in \mathrm{N}}$ converges to 0 .

Lamma 1.3 The sequences $\left\{\mathrm{y}_{2 \mathrm{n}}\right\}_{\mathrm{n} \in \mathrm{N}}$ and $\left\{\mathrm{y}_{2 \mathrm{n}+1}\right\}_{\mathrm{n} \in \mathrm{No}}$ are Cauchy sequences.

Proof : Suppose it is no so, then there exists an $\varepsilon>0$ such that for any integer $2 \mathrm{k}$ there exists two sequences $\{2 \mathrm{~m}(\mathrm{k})\}_{\mathrm{k} \in \mathrm{N}}$ and $\{2 \mathrm{n}(\mathrm{k})\}_{\mathrm{k} \in \mathrm{N}}$ with $2 \mathrm{~m}(\mathrm{k})>2 \mathrm{n}(\mathrm{k})>\mathrm{k}$ for which

$$
\mathrm{d}\left(\mathrm{y}_{2 \mathrm{n}(\mathrm{k})}, \mathrm{y}_{2 \mathrm{~m}(\mathrm{k})}\right)>\varepsilon \text {. }
$$

If $2 \mathrm{~m}(\mathrm{k})$ denotes the smallest integer exceeding $2 \mathrm{n}(\mathrm{k})$ satisfying (9), we have, $\mathrm{d}\left(\mathrm{y}_{2 \mathrm{n}(\mathrm{k})}, \mathrm{y}_{2 \mathrm{~m}(\mathrm{k})-2}\right) \leq \varepsilon$.

Then, $\quad \varepsilon<\mathrm{d}\left(\mathrm{y}_{2 \mathrm{n}(\mathrm{k})}, \mathrm{y}_{2 \mathrm{~m}(\mathrm{k})}\right) \leq \mathrm{d}\left(\mathrm{y}_{2 \mathrm{n}(\mathrm{k})}, \mathrm{y}_{2 \mathrm{~m}(\mathrm{k})-2}\right)+\mathrm{d}_{2 \mathrm{~m}(\mathrm{k})-2}+\mathrm{d}_{2 \mathrm{~m}(\mathrm{k})-1}$

For any even integer $2 \mathrm{k}$ this implies that

$$
\lim _{k \rightarrow \infty} d\left(y_{2 n(k)}, y_{2 m(k)}\right)=\varepsilon
$$

Using (4) and the non decreasing property of $f$, we get

$$
\begin{aligned}
& \mathrm{d}\left(\mathrm{y}_{2 \mathrm{n}(\mathrm{k})}, \mathrm{y}_{2 \mathrm{~m}(\mathrm{k})}\right) \leq \mathrm{d}_{2 \mathrm{n}(\mathrm{k})}+\mathrm{d}\left(\mathrm{y}_{2 \mathrm{n}(\mathrm{k})+1}, \mathrm{y}_{2 \mathrm{~m}(\mathrm{k})}\right) \\
& \leq \mathrm{d}_{2 \mathrm{n}(\mathrm{k})}-\mathrm{bd}_{2 \mathrm{~m}(\mathrm{k})-1}+\min \left\{\mathrm{d}\left(\mathrm{y}_{2 \mathrm{~m}(\mathrm{k})-1}, \mathrm{y}_{2 \mathrm{n}(\mathrm{k})+1}\right), \mathrm{d}_{2 \mathrm{n}(\mathrm{k})}\right\} \\
& +\mathrm{f}\left(\operatorname { m a x } \left\{\mathrm{d}\left(\mathrm{y}_{2 \mathrm{n}(\mathrm{k})}, \mathrm{y}_{2 \mathrm{~m}(\mathrm{k})-1}\right), \mathrm{d}_{2 \mathrm{n}(\mathrm{k})}, \mathrm{d}_{2 \mathrm{~m}(\mathrm{k})-1}, \mathrm{~d}\left(\mathrm{y}_{2 \mathrm{~m}(\mathrm{k})-1}, \mathrm{y}_{2 \mathrm{n}(\mathrm{k})+1}\right),\right.\right. \\
& \left.\left.\left[\mathrm{d}\left(\mathrm{y}_{2 \mathrm{n}(\mathrm{k})}, \mathrm{y}_{2 \mathrm{~m}(\mathrm{k})}\right)+\mathrm{d}\left(\mathrm{y}_{2 \mathrm{~m}(\mathrm{k})-1}, \mathrm{y}_{2 \mathrm{n}(\mathrm{k})+1}\right)\right] / 2\right\}\right) \\
& \leq \mathrm{d}_{2 \mathrm{n}(\mathrm{k})}-\mathrm{bd}_{2 \mathrm{~m}(\mathrm{k})-1}+\min \left\{\mathrm{d}\left(\mathrm{y}_{2 \mathrm{~m}(\mathrm{k})-1}, \mathrm{y}_{2 \mathrm{n}(\mathrm{k})+1}\right), \mathrm{d}_{2 \mathrm{n}(\mathrm{k})}\right\} \\
& +\mathrm{f}\left(\operatorname { m a x } \left\{\mathrm{d}\left(\mathrm{y}_{2 \mathrm{n}(\mathrm{k})}, \mathrm{y}_{2 \mathrm{~m}(\mathrm{k})}\right)+\mathrm{d}_{2 \mathrm{~m}(\mathrm{k})-1}, \mathrm{~d}_{2 \mathrm{n}(\mathrm{k})}, \mathrm{d}_{2 \mathrm{~m}(\mathrm{k})-1}, \mathrm{~d}_{2 \mathrm{~m}(\mathrm{k})-2}+\varepsilon+\mathrm{d}_{2 \mathrm{n}(\mathrm{k})}\right.\right. \text {, } \\
& \left.\left[\mathrm{d}\left(\mathrm{y}_{2 \mathrm{n}(\mathrm{k})}, \mathrm{y}_{2 \mathrm{~m}(\mathrm{k})}\right)+\mathrm{d}_{2 \mathrm{~m}(\mathrm{k})-2}+\varepsilon+\mathrm{d}_{2 \mathrm{n}(\mathrm{k})} / 2\right\}\right)
\end{aligned}
$$

Using Lemma 1.2, (10) and the upper semi continuity of $\mathrm{f}$, we get as $\mathrm{k} \rightarrow \infty$,

$$
\varepsilon \leq \mathrm{f}(\max \{\varepsilon, 0,0, \varepsilon,[\varepsilon+\varepsilon] / 2\})=\mathrm{f}(\varepsilon)
$$

which contradicts the fact that $\mathrm{f}(\varepsilon)<\varepsilon$ as $\varepsilon>0$. Thus $\left\{\mathrm{y}_{2 \mathrm{n}}\right\}_{\mathrm{n} \in \mathrm{N}}$ is a Cauchy sequence. Similarly we can show that $\left\{\mathrm{y}_{2 \mathrm{n}+1}\right\}_{\mathrm{n} \in \mathrm{N}}$ is also a Cauchy sequence. Hence the lemma.

\section{Main Results}

Taking a clue from the result of Sessa et. al. [10], we have the following theorem for a family of mappings.

Theorem 2.1 Let $\mathrm{S}, \mathrm{T}: \mathrm{X} \rightarrow \mathrm{X}$ and either $\mathrm{S}$ or $\mathrm{T}$ be continuous. Then $\mathrm{S}$ and $\mathrm{T}$ have a common fixed point $\mathrm{w}$ if and only if there exists a family $\left\{\mathrm{A}_{\mathrm{i}}\right\}_{\mathrm{i} \in \mathrm{N}}$ of self mappings of $\mathrm{X}$ and $\mathrm{f} \in \mathrm{F}$ satisfying the condition

(vii) $\quad A_{i}(X) \subset S(X) \cap T(X)$, for each $i \in N$

(viii) $\quad A_{i}$ weakly commutes with $S$ and $T$, for each $i \in N$

(ix) $\quad \operatorname{ad}\left(\mathrm{A}_{\mathrm{i}} \mathrm{x}, \mathrm{A}_{\mathrm{j}} \mathrm{y}\right)+\mathrm{bd}\left(\mathrm{Ty}, \mathrm{A}_{\mathrm{j}} \mathrm{y}\right)-\min \left\{\mathrm{d}\left(\mathrm{Ty}, \mathrm{A}_{\mathrm{i}} \mathrm{x}\right), \mathrm{d}\left(\mathrm{Sx}, \mathrm{A}_{\mathrm{i}} \mathrm{x}\right)\right\}$ 


$$
\leq \mathrm{f}\left(\max \left\{\mathrm{d}(\mathrm{Sx}, \mathrm{Ty}), \mathrm{d}\left(\mathrm{Sx}, \mathrm{A}_{\mathrm{i}} \mathrm{x}\right), \mathrm{d}\left(\mathrm{Ty}, \mathrm{A}_{\mathrm{j}} \mathrm{y}\right), \mathrm{d}\left(\mathrm{Ty}, \mathrm{A}_{\mathrm{i}} \mathrm{x}\right),\left[\mathrm{d}\left(\mathrm{Sx}, \mathrm{A}_{\mathrm{j}} \mathrm{y}\right)+\mathrm{d}\left(\mathrm{Ty}, \mathrm{A}_{\mathrm{i}} \mathrm{x}\right)\right] / 2\right\}\right)
$$

for all $x, y \in X, i, j \in N, i \neq j, a, b \geq 0$ with $a \geq 2$. Then $w$ is the unique common fixed point of $\left\{A_{i}\right\}_{i \in N}, S$ and $\mathrm{T}$.

Proof : By Lemma 1.3, the sequence $\left\{\mathrm{y}_{2 \mathrm{n}+1}\right\}_{\mathrm{n} \in \mathrm{No}}$, as defined in (5) converges to a point $\mathrm{w}$, for $\mathrm{X}$ is complete. By Lemma 1.2, we have

$$
0=\lim _{n \rightarrow \infty} d_{2 n}=\lim _{n \rightarrow \infty} d\left(y_{2 n}, y_{2 n+1}\right)=\lim _{n \rightarrow \infty} d\left(y_{2 n}, w\right) .
$$

Thus the sequence $\left\{\mathrm{y}_{2 \mathrm{n}}\right\}$, defined in (6), also converges to w. Now we suppose the continuity of the mapping S. Then

$$
\mathrm{Sw}=\mathrm{S}\left(\lim _{\mathrm{n}} \mathrm{y}_{2 \mathrm{n}+1}\right)=\mathrm{S}\left(\lim _{\mathrm{n} \rightarrow \infty} \mathrm{A}_{2 \mathrm{n}+1} \mathrm{x}_{2 \mathrm{n}}\right)=\lim _{\mathrm{n} \rightarrow \infty} \mathrm{SA}_{2 \mathrm{n}+1} \mathrm{x}_{2 \mathrm{n}}
$$

Since $S$ weakly commutes with $A_{i}$ for any $i \in N$, we have

$$
\begin{aligned}
\mathrm{d}\left(\mathrm{A}_{2 \mathrm{n}+1} \mathrm{Sx}_{2 \mathrm{n}}, \mathrm{Sw}\right) & \leq \mathrm{d}\left(\mathrm{A}_{2 \mathrm{n}+1} \mathrm{Sx}_{2 \mathrm{n}}, \mathrm{SA}_{2 \mathrm{n}+1} \mathrm{x}_{2 \mathrm{n}}\right)+\mathrm{d}\left(\mathrm{SA}_{2 \mathrm{n}+1} \mathrm{x}_{2 \mathrm{n}}, \mathrm{Sw}\right) \\
& \leq \mathrm{d}\left(\mathrm{A}_{2 \mathrm{n}+1} \mathrm{x}_{2 \mathrm{n}}, \mathrm{Sx}_{2 \mathrm{n}}\right)+\mathrm{d}\left(\mathrm{SAx}_{2 \mathrm{n}+1}, \mathrm{Sw}\right)
\end{aligned}
$$

which implies, as $\mathrm{n} \rightarrow \infty$, that the sequence $\left\{\mathrm{A}_{2 \mathrm{n}+1} \mathrm{Sx}_{2 \mathrm{n}}\right\}_{\mathrm{n} \in \mathrm{N}}$ also converge to $\mathrm{Sw}$.

using (vii), we deduce

$$
\begin{aligned}
\operatorname{ad}\left(\mathrm{A}_{2 \mathrm{n}+1} \mathrm{Sx}_{2 \mathrm{n}}, \mathrm{A}_{2 \mathrm{n}} \mathrm{x}_{2 \mathrm{n}-1}\right)+ & \operatorname{bd}\left(\mathrm{Tx}_{2 \mathrm{n}-1}, \mathrm{~A}_{2 \mathrm{n}} \mathrm{x}_{2 \mathrm{n}-1}\right)-\min \left\{\mathrm{d}\left(\mathrm{Tx}_{2 \mathrm{n}-1}, \mathrm{~A}_{2 \mathrm{n}+1} \mathrm{Sx}_{2 \mathrm{n}}\right), \mathrm{d}\left(\mathrm{S}^{2} \mathrm{x}_{2 \mathrm{n}}, \mathrm{A}_{2 \mathrm{n}+1} \mathrm{Sx}_{2 \mathrm{n}}\right)\right\} \\
\leq & \mathrm{f}\left(\operatorname { m a x } \left\{\mathrm{d}\left(\mathrm{S}^{2} \mathrm{x}_{2 \mathrm{n}}, \mathrm{Tx}_{2 \mathrm{n}-1}\right), \mathrm{d}\left(\mathrm{S}^{2} \mathrm{x}_{2 \mathrm{n}}, \mathrm{A}_{2 \mathrm{n}+1} \mathrm{Sx}_{2 \mathrm{n}}\right), \mathrm{d}\left(\mathrm{Tx}_{2 \mathrm{n}-1}, \mathrm{~A}_{2 \mathrm{n}} \mathrm{x}_{2 \mathrm{n}-1}\right),\right.\right. \\
& \left.\left.\mathrm{d}\left(\mathrm{Tx}_{2 \mathrm{n}-1}, \mathrm{~A}_{2 \mathrm{n}+1} \mathrm{Sx}_{2 \mathrm{n}}\right),\left[\mathrm{d}\left(\mathrm{S}^{2} \mathrm{x}_{2 \mathrm{n}}, \mathrm{A}_{2 \mathrm{n}} \mathrm{x}_{2 \mathrm{n}-1}\right)+\mathrm{d}\left(\mathrm{Tx}_{2 \mathrm{n}-1}, \mathrm{~A}_{2 \mathrm{n}+1} \mathrm{Sx}_{2 \mathrm{n}}\right)\right] / 2\right\}\right)
\end{aligned}
$$

which implies, as limit $n \rightarrow \infty$, that

$$
\begin{aligned}
\operatorname{ad}(\mathrm{Sw}, \mathrm{w}) & \leq \mathrm{f}(\max \{\mathrm{d}(\mathrm{Sw}, \mathrm{w}), \mathrm{d}(\mathrm{Sw}, \mathrm{Sw}), \mathrm{d}(\mathrm{w}, \mathrm{w}), \mathrm{d}(\mathrm{w}, \mathrm{Sw}),[\mathrm{d}(\mathrm{Sw}, \mathrm{w})+\mathrm{d}(\mathrm{w}, \mathrm{Sw}) / 2\}) \\
& =\mathrm{f}(\mathrm{d}(\mathrm{Sw}, \mathrm{w}))<\mathrm{d}(\mathrm{Sw}, \mathrm{w})
\end{aligned}
$$

Therefore, $\mathrm{Sw}=\mathrm{w}$.

Using (vii) again, we have for any odd integer $i \in \mathrm{N}$.

$$
\begin{gathered}
\operatorname{ad}\left(\mathrm{A}_{\mathrm{i}} \mathrm{w}, \mathrm{A}_{2 \mathrm{n}} \mathrm{x}_{2 \mathrm{n}-1}\right)+\operatorname{bd}\left(\mathrm{Tx}_{2 \mathrm{n}-1}, \mathrm{~A}_{2 \mathrm{n}} \mathrm{x}_{2 \mathrm{n}-1}\right)-\min \left\{\mathrm{d}\left(\mathrm{Tx}_{2 \mathrm{n}-1}, \mathrm{~A}_{\mathrm{i}} \mathrm{w}\right), \mathrm{d}\left(\mathrm{Sw}, \mathrm{A}_{\mathrm{i}} \mathrm{w}\right)\right\} \\
\leq \mathrm{f}\left(\operatorname { m a x } \left\{\mathrm{d}\left(\mathrm{Sw}, \mathrm{Tx}_{2 \mathrm{n}-1}\right), \mathrm{d}\left(\mathrm{Sw}, \mathrm{A}_{\mathrm{i}} \mathrm{w}\right), \mathrm{d}\left(\mathrm{Tx}_{2 \mathrm{n}-1}, \mathrm{~A}_{2 \mathrm{n}} \mathrm{x}_{2 \mathrm{n}-1}\right), \mathrm{d}\left(\mathrm{Tx}_{2 \mathrm{n}-1}, \mathrm{~A}_{\mathrm{i}} \mathrm{w}\right),\right.\right. \\
\left.\left.\left[\mathrm{d}\left(\mathrm{Sw}, \mathrm{A}_{2 \mathrm{n}} \mathrm{x}_{2 \mathrm{n}-1}\right)+\mathrm{d}\left(\mathrm{Tx}_{2 \mathrm{n}-1}, \mathrm{~A}_{\mathrm{i}} \mathrm{w}\right)\right] / 2\right\}\right)
\end{gathered}
$$

Taking limit as $\mathrm{n} \rightarrow \infty$, we have

$$
\begin{aligned}
& \operatorname{ad}\left(\mathrm{A}_{\mathrm{i}} \mathrm{w}, \mathrm{w}\right)+\mathrm{bd}(\mathrm{w}, \mathrm{w})-\min \left\{\mathrm{d}\left(\mathrm{w}, \mathrm{A}_{\mathrm{i}} \mathrm{w}\right), \mathrm{d}\left(\mathrm{Sw}, \mathrm{A}_{\mathrm{i}} \mathrm{w}\right)\right\} \\
& \leq \mathrm{f}\left(\max \left\{\mathrm{d}(\mathrm{Sw}, \mathrm{w}), \mathrm{d}\left(\mathrm{Sw}, \mathrm{A}_{\mathrm{i}} \mathrm{w}\right), \mathrm{d}(\mathrm{w}, \mathrm{w}), \mathrm{d}\left(\mathrm{w}, \mathrm{A}_{\mathrm{i}} \mathrm{w}\right),\left[\mathrm{d}(\mathrm{Sw}, \mathrm{w})+\mathrm{d}\left(\mathrm{w}, \mathrm{A}_{\mathrm{i}} \mathrm{w}\right) / 2\right\}\right)\right. \\
& \text { or, } \quad \operatorname{ad}\left(\mathrm{A}_{\mathrm{i}} \mathrm{w}, \mathrm{w}\right)-\mathrm{d}\left(\mathrm{A}_{\mathrm{i}} \mathrm{w}, \mathrm{w}\right) \leq \mathrm{f}\left(\max \left\{0, \mathrm{~d}\left(\mathrm{w}, \mathrm{A}_{\mathrm{i}} \mathrm{w}\right), 0, \mathrm{~d}\left(\mathrm{w}, \mathrm{A}_{\mathrm{i}} \mathrm{w}\right), \mathrm{d}\left(\mathrm{w}, \mathrm{A}_{\mathrm{i}} \mathrm{w}\right) / 2\right\}\right) \\
& \text { or, } \quad(\mathrm{a}-1) \mathrm{d}\left(\mathrm{A}_{\mathrm{i}} \mathrm{w}, \mathrm{w}\right) \leq \mathrm{f}\left(\mathrm{d}\left(\mathrm{A}_{\mathrm{i}} \mathrm{w}, \mathrm{w}\right)\right)<\mathrm{d}\left(\mathrm{A}_{\mathrm{i}} \mathrm{w}, \mathrm{w}\right)
\end{aligned}
$$


which gives $\mathrm{w}=\mathrm{A}_{\mathrm{i}} \mathrm{w}$, for any odd integer $\mathrm{i} \in \mathrm{N}$ as $\mathrm{a} \geq 2$.

Now by (vii) we clearly have $w \in \cap_{n \in N} A_{2 n+1}(X) \subseteq T(X)$ and therefore there exists a point $w^{\prime} \in X$ such that $\mathrm{A}_{\mathrm{i}} \mathrm{w}=\mathrm{Tw}^{\prime}=\mathrm{w}$ for any odd integer $\mathrm{i} \in \mathrm{N}$.

Then using (vii) we deduce for any even integer $j \in N$ and for any odd integer $i \in N$.

$$
\begin{aligned}
& \operatorname{ad}\left(\mathrm{A}_{\mathrm{i}} \mathrm{w}, \mathrm{A}_{\mathrm{j}} \mathrm{w}^{\prime}\right)+\mathrm{bd}\left(\mathrm{Tw}^{\prime}, \mathrm{A}_{\mathrm{j}} \mathrm{w}^{\prime}\right)-\min \left\{\mathrm{d}\left(\mathrm{Tw}^{\prime}, \mathrm{A}_{\mathrm{i}} \mathrm{w}\right), \mathrm{d}\left(\mathrm{Sw}, \mathrm{A}_{\mathrm{i}} \mathrm{w}\right)\right\} \\
& \leq \mathrm{f} \max \left\{\mathrm{d}\left(\mathrm{Sw}, \mathrm{Tw^{ \prime }}\right), \mathrm{d}\left(\mathrm{Sw}, \mathrm{A}_{\mathrm{i} w}\right), \mathrm{d}\left(\mathrm{Tw}^{\prime}, \mathrm{A}_{\mathrm{j}} \mathrm{w}^{\prime}\right), \mathrm{d}\left(\mathrm{Tw}^{\prime}, \mathrm{A}_{\mathrm{i}} \mathrm{w}\right),\left[\mathrm{d}\left(\mathrm{Sw}, \mathrm{A}_{\mathrm{j}} \mathrm{w}^{\prime}\right)+\right.\right. \\
& \left.\left.\mathrm{d}\left(\mathrm{Tw}^{\prime}, \mathrm{A}_{\mathrm{i}} \mathrm{w}\right)\right] / 2\right\}
\end{aligned}
$$

or, $\quad \operatorname{ad}\left(\mathrm{w}, \mathrm{A}_{\mathrm{j}} \mathrm{w}^{\prime}\right)+\mathrm{bd}\left(\mathrm{w}, \mathrm{A}_{\mathrm{j}} \mathrm{w}^{\prime}\right)-\min \{\mathrm{d}(\mathrm{w}, \mathrm{w}), \mathrm{d}(\mathrm{w}, \mathrm{w})\}$

$$
\leq \mathrm{fmax}\left\{\mathrm{d}(\mathrm{w}, \mathrm{w}), \mathrm{d}(\mathrm{w}, \mathrm{w}), \mathrm{d}\left(\mathrm{w}, \mathrm{A}_{\mathrm{j}} \mathrm{w}^{\prime}\right), \mathrm{d}(\mathrm{w}, \mathrm{w}),\left[\mathrm{d}\left(\mathrm{w}, \mathrm{A}_{\mathrm{j}} \mathrm{w}^{\prime}\right)+\mathrm{d}(\mathrm{w}, \mathrm{w})\right] / 2\right\}
$$

Hence $\quad(a+b) d\left(w, A_{j} w^{\prime}\right) \leq f\left(d\left(w, A_{j} w^{\prime}\right)\right)<d\left(w, A_{j} w^{\prime}\right)$,

which implies that $A_{j} w^{\prime}=w$, for any even integer $j \in N$. $A_{k}$ being weakly commuting with $T$ for any $k \in N$, we have

$$
d\left(T A_{j} w^{\prime}, A_{j} T w^{\prime}\right) \leq d\left(T w^{\prime}, A_{j} w^{\prime}\right)=d(w, w)=0
$$

and hence

$$
\mathrm{Tw}=\mathrm{TA}_{\mathrm{j}} \mathrm{w}^{\prime}=\mathrm{A}_{\mathrm{j}} \mathrm{Tw}^{\prime}=\mathrm{A}_{\mathrm{j}} \mathrm{w} .
$$

for any even integer $\mathrm{j} \in \mathrm{N}$. Moreover (viii) implies for any odd integer $\mathrm{i} \in \mathrm{N}$ and for any even integer $\mathrm{j}$ $\in \mathrm{N}$.

$$
\begin{aligned}
& \operatorname{ad}\left(\mathrm{A}_{\mathrm{i}} \mathrm{w}, \mathrm{A}_{\mathrm{j}} \mathrm{w}\right)+\mathrm{bd}\left(\mathrm{Tw}, \mathrm{A}_{\mathrm{j}} \mathrm{w}\right)-\min \left\{\mathrm{d}\left(\mathrm{Tw}, \mathrm{A}_{\mathrm{i}} \mathrm{w}\right), \mathrm{d}\left(\mathrm{Sw}, \mathrm{A}_{\mathrm{i}} \mathrm{w}\right)\right\} \\
& \leq \mathrm{f}\left(\max \left\{\mathrm{d}(\mathrm{Sw}, \mathrm{Tw}), \mathrm{d}\left(\mathrm{Sw}, \mathrm{A}_{\mathrm{i}} \mathrm{w}\right), \mathrm{d}\left(\mathrm{Tw}, \mathrm{A}_{\mathrm{j}} \mathrm{w}\right), \mathrm{d}\left(\mathrm{Tw}, \mathrm{A}_{\mathrm{i}} \mathrm{w}\right),\left[\mathrm{d}\left(\mathrm{Sw}, \mathrm{A}_{\mathrm{j}} \mathrm{w}\right)+\mathrm{d}\left(\mathrm{Tw}, \mathrm{A}_{\mathrm{i}} \mathrm{w}\right)\right] / 2\right\}\right) \\
& \text { or, } \operatorname{ad}(\mathrm{w}, \mathrm{Tw})+\mathrm{bd}(\mathrm{Tw}, \mathrm{Tw})-\min \{\mathrm{d}(\mathrm{Tw}, \mathrm{w}), \mathrm{d}(\mathrm{w}, \mathrm{w})\} \\
& \leq f\left(\operatorname { m a x } \left\{d(S w, T w), d\left(S w, A_{i} w\right), d\left(T w, A_{j} w\right), d\left(T w, A_{i} w\right),\left[d\left(S w, A_{j} w\right)+\right.\right.\right. \\
& \left.\left.\left.\mathrm{d}\left(\mathrm{Tw}, \mathrm{A}_{\mathrm{i}} \mathrm{w}\right)\right] / 2\right\}\right)
\end{aligned}
$$

Thus, $\quad \operatorname{ad}(w, T w) \leq f(\max \{d(w, T w), 0,0, d(T w, w), d(w, T w)\})$

i.e. $\quad a d(w, T w) \leq f(d(w, T w))<d(w, T w)$.

Thus $w=T w$ and so $A_{j} w=w$ for any even integer $j \in N$. Therefore $w$ is a common fixed point of $\left\{\mathrm{A}_{\mathrm{i}}\right\}_{\mathrm{i} \in \mathrm{N}}, \mathrm{S}$ and $\mathrm{T}$.

Similar proof can be exhibited if one supposes the continuity of T instead of S. Further from (vii) it is easily seen that $\mathrm{w}$ is unique common fixed point of $\left\{\mathrm{A}_{\mathrm{i}}\right\}_{\mathrm{i} \in \mathrm{N}}, \mathrm{S}$ and $\mathrm{T}$. This concludes the sufficient part of the proof.

To show the necessary of the condition, let $\mathrm{w}$ be a common fixed point of $\mathrm{S}$ and $\mathrm{T}$. Define $A_{i}(x)=w$ for any $i \in N$ and for any $x \in X$. Now since $d\left(A_{i} x, A_{j} y\right)=d(w, w)=0$ for any 
$\mathrm{i}, \mathrm{j} \in \mathrm{N}, \mathrm{i} \neq \mathrm{j}$ and for any $\mathrm{x}, \mathrm{y} \in \mathrm{X}$. It is trivial that (x) holds for any function $\mathrm{f} \in \mathrm{F}$ and further (xiv) is verified being

$$
\{\mathrm{w}\}=\mathrm{A}_{\mathrm{i}}(\mathrm{x}) \subseteq \mathrm{S}(\mathrm{X}) \cap \mathrm{T}(\mathrm{X})
$$

for any $i \in N$. Clearly $w$ is the unique common fixed point of $\left\{A_{i}\right\}_{i \in N}, S$ and $T$. This completes the proof.

Using weak commutativity of mappings we have an extension of Theorem 1.2 Chang [2] as follows.

Theorem 2.2 Let $\mathrm{S}, \mathrm{T}: \mathrm{X} \rightarrow \mathrm{X}$ and either $\mathrm{S}$ or $\mathrm{T}$ be continuous. Then $\mathrm{S}$ and $\mathrm{T}$ have a common fixed point $w$ if and only if there exists two self mappings $A, B$ of $X$ and a function $f \in F$ such that

(x) $\mathrm{A}(\mathrm{X}) \cup \mathrm{B}(\mathrm{X}) \subseteq \mathrm{S}(\mathrm{X}) \cap \mathrm{T}(\mathrm{X})$.

(xi) both $\mathrm{A}$ and $\mathrm{B}$ weakly commute with $\mathrm{S}$ and $\mathrm{T}$.

(xii) $\mathrm{d}(\mathrm{Ax}, \mathrm{By}) \leq \mathrm{f}(\max \{\mathrm{d}(\mathrm{Sx}, \mathrm{Ty}), \mathrm{d}(\mathrm{Sx}, \mathrm{Ax}), \mathrm{d}(\mathrm{Ty}, \mathrm{By}),[\mathrm{d}(\mathrm{Sx}, \mathrm{By})+\mathrm{d}(\mathrm{Ty}, \mathrm{Ax})] / 2\})$

Further $\mathrm{w}$ is the unique common fixed point of $\mathrm{A}, \mathrm{B}, \mathrm{S}$ and $\mathrm{T}$.

As the proof goes in a similar manner as of Theorem 2.1, so we omit it. However we give an example in order to show that the Theorem 2.2 is stronger result than Theorems 1.2.

Example 2.1 Let $\mathrm{X}=[0,1]$ with Euclidean metric.

Define $\quad \mathrm{Ax}=\frac{x}{\mathrm{a}}-\frac{z^{2}}{34}, \mathrm{Bx}=\frac{x}{4}-\frac{z^{2}}{12}, \mathrm{Sx}=\frac{x}{2}, \mathrm{Tx}=\mathrm{x}$ for any $\mathrm{x} \in \mathrm{X}$.

we have

$$
\begin{aligned}
& \mathrm{ASx}=\mathrm{A}\left(\frac{z}{2}\right)=\frac{z}{16}-\frac{z^{2}}{286} \text { and } \mathrm{SAx}=\mathrm{S}\left(\frac{z}{8}-\frac{z^{2}}{64}\right)=\frac{z}{15}-\frac{z^{2}}{128} \\
& \mathrm{~d}(\mathrm{ASx}, \mathrm{SAx})=\left(\frac{x}{26}-\frac{x^{2}}{286}-\frac{x}{16}+\frac{x^{2}}{128}\right) \\
& =\frac{x^{2}}{286} \leq \frac{8 x}{8}+\frac{z^{2}}{64}=\frac{z}{2}-\left(\frac{z}{8}-\frac{w^{2}}{64}\right)=\mathrm{d}(\mathrm{Sx}, \mathrm{Ax}) \quad \text { for any } \mathrm{x} \in \mathrm{X} \text {. }
\end{aligned}
$$

Thus S weakly commute with $\mathrm{A}$, but $\mathrm{S}$ and $\mathrm{A}$ do not commute being

$$
\mathrm{ASx}=\frac{z}{16}-\frac{z^{2}}{286} \neq \frac{z}{16}-\frac{w^{2}}{128}=\mathrm{SAx} \text { for any } \mathrm{x} \in(0,1] \text {. }
$$

Moreover $\mathrm{B}$ commutes with $\mathrm{T}$. Let $\mathrm{F}$ be the set of all real function $\mathrm{f}:[0, \infty) \rightarrow[0, \infty)$ such that for every $\mathrm{f} \in \mathrm{F}$ is non decreasing, upper semi continuous and $\mathrm{f}(\mathrm{t})<\mathrm{t}$ for any $\mathrm{t}>0$ and let

Then we have

$$
\begin{aligned}
f(t) & =\frac{1}{4} t-\frac{1}{16} t^{2} \quad \text { if } 0 \leq t \leq 1 \\
& =\frac{3}{8} t \quad \text { if } t>1 .
\end{aligned}
$$

$$
\mathrm{d}(\mathrm{Ax}, \mathrm{By})=\left|\frac{z}{8}-\frac{z^{2}}{64}-\frac{y}{4}+\frac{y^{2}}{16}\right|
$$




$$
\begin{aligned}
& =\left|\left(\frac{x}{0}-\frac{y}{4}\right)-\frac{1}{16}\left(\frac{1}{4} x^{2}-y^{2}\right)\right| \\
& =\frac{1}{4}\left|\frac{1}{2} x-y\right|\left[1-\frac{1}{4}\left(\frac{1}{2} x+y\right)\right] \\
& \leq \frac{1}{4}\left|\frac{1}{2} x-y\right|\left[1-\frac{1}{4}\left|\frac{1}{2} x-y\right|\right] \\
& \leq \frac{1}{4}\left|\frac{1}{2} x-y\right|-\frac{1}{16}\left|\frac{1}{2} x-y\right|^{2} \\
& =f(d(\text { Sx, Ty })) \\
& \quad \quad \quad f\left(\max \left\{\mathrm{d}(\mathrm{Sx}, \mathrm{Ty}), \mathrm{d}(\mathrm{Sx}, \mathrm{Ax}), \mathrm{d}(\mathrm{Ty}, \mathrm{By}), \frac{1}{2}[\mathrm{~d}(\mathrm{Sx}, \mathrm{By})+\mathrm{d}(\mathrm{Ty}, \mathrm{Ax})]\right\}\right)
\end{aligned}
$$

for any $x, y \in X$. Further,

$$
\begin{aligned}
\mathrm{A}(\mathrm{X}) \cup \mathrm{B}(\mathrm{X})=\left[0, \frac{7}{64}\right] \cup\left[0, \frac{3}{15}\right] & \\
=\left[0, \frac{5}{15}\right] \subset\left[0, \frac{1}{2}\right] & =\left[0, \frac{1}{2}\right] \cap[0,1] \\
& =\mathrm{S}(\mathrm{X}) \cap \mathrm{T}(\mathrm{X}) .
\end{aligned}
$$

Thus we see that all the assumptions of the Theorem 2.2 are verified and 0 is the unique common fixed point, whereas Theorems 1.1 and 1.2 are not applicable because the mappings $\mathrm{S}$ and $\mathrm{A}$ do not commute.

Theorem 2.3 Let $\mathrm{T}_{1}$ and $\mathrm{T}_{2}$ be two continuous mappings of a complete metric space $(\mathrm{X}, \mathrm{d})$ into itself such that

$$
\mathrm{d}\left(\mathrm{T}_{1} \mathrm{x}, \mathrm{T}_{2} \mathrm{y}\right)<\max \left\{\mathrm{d}(\mathrm{x}, \mathrm{y}), \mathrm{d}\left(\mathrm{x}, \mathrm{T}_{1} \mathrm{x}\right)\right\}
$$

and there exists a subset $\mathrm{A} \subset \mathrm{X}$ and a point $\mathrm{x}_{0} \in \mathrm{A}$ satisfying the following conditions :

$$
\mathrm{d}\left(\mathrm{x}_{0}, \mathrm{~T}_{\mathrm{i}} \mathrm{x}\right)-\mathrm{d}\left(\mathrm{T}_{1} \mathrm{x}_{0}, \mathrm{~T}_{1} \mathrm{~T}_{2} \mathrm{x}\right) \geq 2 \mathrm{~d}\left(\mathrm{x}_{0}, \mathrm{~T}_{1} \mathrm{x}_{0}\right),
$$

for $\mathrm{x}, \mathrm{y}$ in $\mathrm{X}-\mathrm{A}, \mathrm{i}=1,2$ and $\mathrm{T}_{1} \mathrm{~T}_{2}=\mathrm{T}_{2} \mathrm{~T}_{1}$.

$$
d\left(T_{1} x, T_{2} y\right) \leq \alpha d(x, y)\left\{d\left(x, T_{1} x\right) d\left(y, T_{2} y\right)\right\}^{1 / 2}
$$

for $\mathrm{x}, \mathrm{y}$ in $\mathrm{A}$, where $\alpha$ is a monotonically decreasing function from $[0, \infty)$ into $[0,1)$. Then there exists a unique common fixed point of $\mathrm{T}_{1}$ and $\mathrm{T}_{2}$.

Proof: Suppose that $x_{0} \neq T_{1} x_{0}$ and define a sequence $\left\{x_{n}\right\}$ of elements $x_{n} \in X$,

$$
T_{1} x_{0}=x_{1}, T_{2} x_{1}=x_{2}, \ldots, T_{1} x_{2 n}=x_{2 n+1}, T_{2} x_{2 n+1}=x_{2 n+2} .
$$

we have

$$
\mathrm{d}\left(\mathrm{T}_{1} \mathrm{x}, \mathrm{T}_{2} \mathrm{y}\right)<\max \left\{\mathrm{d}(\mathrm{x}, \mathrm{y}), \mathrm{d}\left(\mathrm{x}, \mathrm{T}_{1} \mathrm{x}\right)\right\}
$$

Hence,

$$
\mathrm{d}\left(\mathrm{x}_{0}, \mathrm{x}_{2 \mathrm{n}+1}\right)<\max \left\{\mathrm{d}\left(\mathrm{x}_{0}, \mathrm{x}_{1}\right), \mathrm{d}\left(\mathrm{x}_{0}, \mathrm{x}_{0}\right)\right\}=\mathrm{d}\left(\mathrm{x}_{0}, \mathrm{x}_{1}\right) .
$$

From the triangle inequality and $\mathrm{T}_{1} \mathrm{~T}_{2}=\mathrm{T}_{2} \mathrm{~T}_{1}$ we get,

$$
\begin{aligned}
\mathrm{d}\left(\mathrm{x}_{0}, \mathrm{x}_{2 \mathrm{n}+1}\right) & \leq \mathrm{d}\left(\mathrm{x}_{0}, \mathrm{x}_{1}\right)+\mathrm{d}\left(\mathrm{x}_{1}, \mathrm{x}_{2 \mathrm{n}+2}\right)+\mathrm{d}\left(\mathrm{x}_{2 \mathrm{n}+2,}, \mathrm{x}_{2 \mathrm{n}+1}\right) \\
& =\mathrm{d}\left(\mathrm{x}_{0}, \mathrm{x}_{1}\right)+\mathrm{d}\left(\mathrm{T}_{1} \mathrm{x}_{0}, \mathrm{~T}_{2} \mathrm{~T}_{1} \mathrm{x}_{2 \mathrm{n}}\right)+\mathrm{d}\left(\mathrm{x}_{2 \mathrm{n}+2}, \mathrm{x}_{2 \mathrm{n}+1}\right)
\end{aligned}
$$




$$
<2 \mathrm{~d}\left(\mathrm{x}_{0}, \mathrm{x}_{1}\right)+\mathrm{d}\left(\mathrm{T}_{1} \mathrm{x}_{0}, \mathrm{~T}_{2} \mathrm{~T}_{1} \mathrm{x}_{2 \mathrm{n}}\right)
$$

Thus,

$$
\mathrm{d}\left(\mathrm{x}_{0}, \mathrm{~T}_{1} \mathrm{x}_{2 \mathrm{n}}\right)-\mathrm{d}\left(\mathrm{T}_{1} \mathrm{x}_{0}, \mathrm{~T}_{2} \mathrm{~T}_{1} \mathrm{x}_{2 \mathrm{n}}\right)<2 \mathrm{~d}\left(\mathrm{x}_{0}, \mathrm{~T}_{1} \mathrm{x}_{0}\right) .
$$

Hence from the condition ( $\mathrm{xx}$ ), it follows that $\mathrm{x}_{2 \mathrm{n}+1} \in \mathrm{A}$ for every $\mathrm{n}$.

Similarly $\mathrm{x}_{2 \mathrm{n}+2} \in \mathrm{A}$ for all $\mathrm{n}$. Therefore $\mathrm{x}_{\mathrm{n}} \in \mathrm{A}$ for each $\mathrm{n}$.

Next we show that the sequence $\left\{\mathrm{x}_{\mathrm{n}}\right\}$ is bounded. For this consider,

$$
\begin{aligned}
\mathrm{d}\left(\mathrm{x}_{0}, \mathrm{x}_{2(\mathrm{n}+1)}\right) \leq \mathrm{d}\left(\mathrm{x}_{0},\right. & \left.\mathrm{T}_{1} \mathrm{x}_{0}\right)+\mathrm{d}\left(\mathrm{T}_{1} \mathrm{x}_{0}, \mathrm{~T}_{2} \mathrm{x}_{2 \mathrm{n}-1}\right)+\mathrm{d}\left(\mathrm{T}_{1} \mathrm{x}_{2 \mathrm{n}}, \mathrm{T}_{2} \mathrm{x}_{2 \mathrm{n}-1}\right)+\mathrm{d}\left(\mathrm{x}_{2 \mathrm{n}+1}, \mathrm{x}_{2 \mathrm{n}+2}\right) \\
& \leq 3 \mathrm{~d}\left(\mathrm{x}_{0}, \mathrm{~T}_{1} \mathrm{x}_{0}\right)+\alpha\left(\mathrm{d}\left(\mathrm{x}_{0}, \mathrm{x}_{2 \mathrm{n}-1}\right)\right)\left\{\mathrm{d}\left(\mathrm{x}_{0}, \mathrm{x}_{1}\right) \mathrm{d}\left(\mathrm{x}_{2 \mathrm{n}-1}, \mathrm{x}_{2 \mathrm{n}}\right)\right\}^{1 / 2} \\
& \leq\left\{3+\alpha \mathrm{d}\left(\mathrm{x}_{0}, \mathrm{x}_{2 \mathrm{n}-1}\right)\right\} \mathrm{d}\left(\mathrm{x}_{0}, \mathrm{x}_{1}\right)
\end{aligned}
$$

Hence for a given $\mathrm{d}_{0}>0$ with $\mathrm{d}\left(\mathrm{x}_{0}, \mathrm{x}_{2 \mathrm{n}-1}\right) \geq \mathrm{d}_{0}$, we get

$$
\mathrm{d}\left(\mathrm{x}_{0}, \mathrm{x}_{2 \mathrm{n}+2}\right) \leq\left\{3+\alpha \mathrm{d}_{0}\right\} \mathrm{d}\left(\mathrm{x}_{0}, \mathrm{x}_{1}\right) \text {. }
$$

Similarly we can show that

$$
\mathrm{d}\left(\mathrm{x}_{0}, \mathrm{x}_{2 \mathrm{n}+1}\right) \leq\left\{2+\alpha \mathrm{d}_{0}{ }^{*}\right\} \mathrm{d}\left(\mathrm{x}_{0}, \mathrm{x}_{1}\right), \text { for given } \mathrm{d}_{0}{ }^{*}>0, \mathrm{~d}\left(\mathrm{x}_{0}, \mathrm{x}_{2 \mathrm{n}+1}\right) \geq \mathrm{d}_{0}{ }^{*}
$$

and hence $\left\{\mathrm{x}_{\mathrm{n}}\right\}$ is bounded. By routine calculation, it follows that for each $\mathrm{n}$,

$$
d\left(x_{n}, x_{n+1}\right) \leq\left\{\beta\left(d\left(x_{n-1}, x_{n}\right)\right), \beta\left(d\left(x_{n-2}, x_{n-1}\right)\right), \ldots, \beta\left(d\left(x_{0}, x_{1}\right)\right)\right\} d\left(x_{0}, x_{1}\right) \text {, where } \beta=\alpha^{2} .
$$

Let $\varepsilon>0$. If $\mathrm{d}\left(\mathrm{x}_{\mathrm{i}}, \mathrm{x}_{\mathrm{i}+1}\right) \geq \varepsilon$ for $\mathrm{i}=0,1,2, .$. , then

$$
\beta\left(\mathrm{d}\left(\mathrm{x}_{\mathrm{i}}, \mathrm{x}_{\mathrm{i}+1}\right)\right) \leq \beta(\varepsilon)
$$

for $\mathrm{i}=0,1,2, \ldots$ and also $0 \leq \beta(\mathrm{t})<1$. Therefore

$$
\mathrm{d}\left(\mathrm{x}_{\mathrm{n}}, \mathrm{x}_{\mathrm{n}+1}\right) \leq(\beta(\varepsilon))^{\mathrm{n}} \mathrm{d}\left(\mathrm{x}_{0}, \mathrm{x}_{1}\right) .
$$

This proves that $\left\{\mathrm{x}_{\mathrm{n}}\right\}$ is a Cauchy sequence. As $\mathrm{X}$ is complete, $\lim _{\mathrm{n} \rightarrow \infty} \mathrm{X}_{\mathrm{n}}=\xi \in \mathrm{X}$. Using continuity of $\mathrm{T}_{1}$ and $\mathrm{T}_{2}$, we find that $\xi$ is a common fixed point of $\mathrm{T}_{1}$ and $\mathrm{T}_{2}$. Uniqueness of $\xi$ is obvious.

Putting $\mathrm{A}=\mathrm{X}$ in Theorem 2.3, we get the following corollary.

Corollary 2.1 If $\mathrm{X}$ is a complete metric space and $\mathrm{T}_{1}, \mathrm{~T}_{2}$ satisfy

$$
\mathrm{d}\left(\mathrm{T}_{1} \mathrm{x}, \mathrm{T}_{2} \mathrm{y}\right) \leq \alpha \mathrm{d}(\mathrm{x}, \mathrm{y})\left\{\mathrm{d}\left(\mathrm{x}, \mathrm{T}_{1} \mathrm{x}\right) \mathrm{d}\left(\mathrm{y}, \mathrm{T}_{2} \mathrm{y}\right\}^{1 / 2}\right.
$$

for all $\mathrm{x}, \mathrm{y}$ in $\mathrm{X}$. Then $\mathrm{T}_{1}$ and $\mathrm{T}_{2}$ have a unique common fixed point.

Remark 2.1 The inequality condition (4) is more general than the inequality condition of Chang [2] and Sessa et. al. [10] and only one of S and T is continuous than that in Chang [2], where both the mappings were continuous. So our result generalizes the result of Chang in two ways. 


\section{References :}

[1] Shih-sen Chang, Math. Japonica, 26, p121 (1981).

[2] _ _ Math. Japonica, 29, p527 (1984).

[3] B. Fisher, Bull. Inst. Math. Acad. Sinica, 9, p399 (1981).

[4] O. Hadzic, Math. Japonica, 29, p287 (1984).

[5] S. A. Hussain and V. M. Sehgal, Math. Japonica, 26, p287 (1981).

[6] K. Iseki, Bull. Austral. Math. Soc., 10, p75 (1974).

[7] M. S. Khan and M. Imdad, Glasnik Math.,18 (38), p321 (1983).

[8] _ Comm. Fac. Sci. Univ. Ankara, 32, p105 (1983).

[9] B. A. Meade, B.A and Singh, S.P., Bull.Aus.Math.Soc., 16, p49 (1977).

[10] S. Sessa R.N. Mukherjee and T. Som, Math. Japonica, 31(2), p235 (1986).

[11] S. L. Singh, Math. Sem. Notes Kobe Univ., 5, p131 (1977).

[12] S. L. Singh and B. M. L. Tiwari, Proc. Nat. Acad. Sci. Indian Sec. A 51(I), p41(1981). 